chromatography. Unbound neutral oligosaccharides were collected from the flow-through and $\mathrm{Q}$-Sepharose bound acidic polysaccharide fractions were eluted stepwise with $0.25,0.5$ and $1 \mathrm{M} \mathrm{NaCl}$. All fractions were desalted on PD MidiTrap G10 mini-columns. Purified fractions were assessed for inhibitory action on C. difficile 027A, ETEC C410 and S. Typhimurium adherence to human and porcine epithelial cell monolayers in the presence of plantain NSP and its fractions (30 min pre-treatment prior to infection), at MOI 100 for $90 \mathrm{~min}$ (for ETEC and Salmonella) or $120 \mathrm{~min}$ (for C. difficile). Adherent colony forming units (CFU) were enumerated by overnight growth on LB-agar.

Results As observed for plantain NSP, at $5 \mathrm{mg} / \mathrm{mL}$, a Q-Sepharosepurified acidic polysaccharide fraction of soluble plantain NSP also significantly blocked adhesion to human Caco2 colonocytes of gut pathogens $C$. difficile, by $52.3 \pm 7.3 \%$; ETEC, by $74.8 \pm 15.1$; and $S$. Typhimurium, by $92.1 \pm 2.91 \%$ (all $\mathrm{P}<0.001$ Kruskal-Wallis; $\mathrm{N}=2$ experiments, each with $\mathrm{n}=3$ replicates). Conversely, pre-treatment of $\mathrm{Caco} 2$ cells with the neutral polysaccharide fraction of plantain NSP showed little ability to reduce adhesion of $C$. difficile $(10.2 \%$ inhibition) and a reduced ability to inhibit interaction of Salmonella or ETEC (46.6\% and $39.1 \%$ inhibition respectively). Similalrly, acidic fractions of plantain NSP at $5 \mathrm{mg} / \mathrm{mL}$ also blocked adhesion of $\mathrm{S}$. Typhimurium to porcine enterocytic cell-line B10XI compared to untreated control ( $N=4, \mathrm{n}=4 ; P<0.01$ Kruskal-Wallis).

Conclusion Our findings indicate that the inhibitory activity of plantain NSP against diarrhoeal pathogen interaction with the host intestinal epithelium lies in its acidic (pectic) polysaccharide component. Disruption of bacterial-epithelial adherence to the intestinal mucosa by soluble plant fibres, acting as 'contrabiotics', may prove to be of therapeutic benefit.

Disclosure of Interest H. Simpson: None Declared, B. Parsons: None Declared, J. Rhodes Grant/Research Support from: Provexis plc (UK), Consultant for: A member of advisory boards for Atlantic, Proctor and Gamble, Falk, Conflict with: Lecture fees received from Abbott, Falk, Ferring, Glaxo Smith Kline, Proctor and Gamble, Schering Plough, Shire, Wyeth. With the University of Liverpool and Provexis UK, holds a patent for use of soluble plantain fibre as maintenance therapy for Crohn's disease plus a patent pending for its use in antibiotic associated diarrhoea, B. Campbell Grant/ Research Support from: Provexis plc. and Shire, Consultant for: Amgen Inc.

\section{PWE-181 THE DEVELOPMENT AND EVOLUTION OF NUTRITION SERVICE PROVISION IN THE NORTH EAST: 2003-2012}

doi:10.1136/gutjnl-2013-304907.469

1."J H Adam, 'N Thompson. 'Gastroenterology, Freeman Hospital, Newcastle Upon Tyne, Newcastle, UK

Introduction Malnutrition is well recognised as a treatable cause and result of ill-health. The recognition and management of malnutrition hinges upon the quality of organisation of nutrition services as well as the quality of the interventions themselves. Malnutrition is common amongst inpatients and decisions regarding management are often complex. The care of patients with complex nutritional needs can be optimised by combining the expertise of those from multiple allied disciplines. These principles are highlighted in the 2006 NICE guideline: Nutrition Support in Adults. This study analyses the progress made at an organisational level in providing nutrition services in hospitals across the North East of England, with reference to recommendations made by NICE.

Methods Nutrition service provision was evaluated by distributing questionnaires to all acute hospitals in the North East region in 2003, 2007 and 2012. Questionnaires were completed by a dietician from each hospital. The questionnaire addressed the provision of Nutrition Support Teams. We analysed the representation of team members from different disciplines, frequency of meetings and topics discussed.
We also sought qualitative and quantitative data regarding inpatient and outpatient use of Total Parenteral Nutrition (TPN), the use of malnutrition screening tools and access to obesity services.

Results Response rates were $92 \%$ in 2003 and $100 \%$ in 2007 and 2012. Over 9 years the percentage of hospitals with Nutrition Support Teams has increased from 19\% to 42\%. Each team discusses an increasing number of patients as well evaluating and developing their local policies and procedures. In line with NICE recommendations, $100 \%$ of acute trusts have a Nutrition Steering Committee in 2012, rising from $31 \%$ in 2003 and $94 \%$ in 2007 . The number of patients able to access inpatient TPN has increased across the region whilst the provision of home TPN has been concentrated towards larger, tertiary facilities. The use of standardised inpatient screening tools has increased from $80 \%$ in 2003 to $100 \%$ in 2012 . Screening of outpatients in acute hospitals has increased from $15 \%$ in 2003 to $33 \%$ in 2012 .

Conclusion Nutrition service provision has evolved beyond recognition over the last 9 years in the North East. Significant progress has been made in forming focused Nutrition Support Teams, expanding TPN delivery and detecting malnutrition, particularly among the inpatient population. Areas for improvement include the development and promotion of efficient and practical methods to screen outpatients for malnutrition. Ultimately, it is hoped that we can demonstrate our developments have had a significant and sustained impact upon patient care and outcomes.

Disclosure of Interest None Declared.

\section{REFERENCE}

1. Nutrition support in adults (CG32): 2006 NICE guideline

\section{PWE-182 MORTALITY AFTER PEG: REVIEW OF 259 PEG PROCEDURES AT A DISTRICT GENERAL HOSPITAL OVER A 7 YEAR PERIOD}

doi:10.1136/gutjnl-2013-304907.470

1,"K Thavanesan, 'K Barnett, 'S Harding, 'D Hartley, 'S Al-Shamma, 'S A Weaver, 'S D McLaughlin. 'Gastroenterology, Royal Bournemouth and Christchurch Hospital, Bournemouth, UK

Introduction Percutaneous Endoscopic Gastrostomy (PEG) is commonly performed in patients that require medium to long-term enteral feeding. In 2004 NCEPOD reviewed the 30day mortality and rate of complications following therapeutic endoscopy. They reported a $6 \% 30$ day mortality and made recommendations regarding changes in practise. Nationally there is little published data on the 1 year mortality following this procedure. In 2010 we introduced changes to our practise including: 1 . The review of all PEG referrals by a nutrition team, 2. Stringently avoiding PEG for 2 weeks following stroke, 3 . Avoiding PEG in patients with pneumonia or other inter-current illness.

Aim to review the 30day and 1 year mortality following PEG at our institution since the NCEPOD publication.

Methods A prospective endoscopy database is maintained at our institution (HICCS). We searched the database for primary PEG procedures during the period of Jan 2005 to September 2012 and reviewed electronic medical records to establish the overall 30 day and 1 year mortality, and also mortality by indication.

Results A total of 259 procedures were performed. Indications were: Stroke $n=130$ (50\%), Multiple sclerosis (MS) $n=28(10.85 \%)$, Motor neurone disease (MND) $\mathrm{n}=19(7.36 \%)$. Mortality data is given in table 1 .

Overall 30 day mortality by indication was: Stroke $10.8 \%, \mathrm{MND}$, $6.3 \%$, MS $12.5 \%$

1 year mortality: Stroke $31.27 \%$, MND 50.0\%, MS 30.7\%.

Conclusion Stroke is the commonest indication for PEG at our hospital. The mean annual 30day mortality following PEG at our hospital is above that reported by NCEPOD but has fallen considerably since 2010 suggesting that changes in our practise have been 


\begin{tabular}{lllll}
\hline \multicolumn{6}{l}{ Abstract PWE- 182 Table 1 } & & \\
\hline Year & 30 Day Mortality & & 1 Year Mortality \\
\hline 2005 & 11 & $21.6 \%$ & 31 & $60.8 \%$ \\
2006 & 4 & $13.3 \%$ & 12 & $40.0 \%$ \\
2007 & 4 & $13.3 \%$ & 13 & $43.3 \%$ \\
2008 & 0 & $0.0 \%$ & 7 & $36.8 \%$ \\
2009 & 4 & $11.8 \%$ & 14 & $41.2 \%$ \\
2010 & 6 & $15.4 \%$ & 18 & $46.2 \%$ \\
2011 & 1 & $3.8 \%$ & 5 & $19.2 \%$ \\
2012 & 2 & $6.7 \%$ & 6 & $20.0 \%$ \\
Mean & 4 & $10.7 \%$ & 13.25 & $38.4 \%$ \\
\hline
\end{tabular}

effective. 1 year mortality in MND patients is high and emphasises the importance of careful patient selection.

We recommend that other institutions with a high 30 day mortality following PEG adopt the changes in practise outlined above. Ongoing audit of our practise is required.

Disclosure of Interest None Declared.

\section{PWE-183 DOES INSERTION OF A GASTROSTOMY CONFER ANY QUALITY OF LIFE BENEFIT TO EITHER PATIENTS OR THEIR CARERS?}

doi:10.1136/gutjnl-2013-304907.471

1." M Kurien, 'M E McAlindon, ' $\mathrm{J}$ Grant, ' $\mathrm{E} F \mathrm{~F}$ Wong, ${ }^{1} \mathrm{~A}$ J Johnston, ${ }^{2} \mathrm{~B}$ Hoeroldt, ${ }^{3} \mathrm{~K} \mathrm{~L}$ Dear, 'D S Sanders. 'Department of Gastroenterology, Royal Hallamshire Hospital, Sheffield; ' ${ }^{2}$ Department of Gastroenterology, Rotherham Hospital, Rotherham; ${ }^{3}$ Department of Gastroenterology, Chesterfield Royal Hospital, Chesterfield, UK

Introduction Gastrostomy feeding is an effective means of providing enteral nutrition to patients who have functionally normal gastrointestinal tracts but who cannot meet their nutritional requirements because of an inadequate oral intake. Whilst improvements in outcome measures such as nutritional status and mortality have been demonstrated in certain patients undergoing gastrostomy insertion, there remains a paucity of work evaluating another important health outcome measure, which is health related quality of life (HRQoL). Furthermore no previous study has looked at the impact on carers' HRQoL when considering all referral indications for gastrostomy. This prospective, multicenter study evaluates HRQoL in both gastrostomy patients and their carers, with comparisons made with a population control group.

Methods 61 patients (mean age 68 years) and 58 carers (mean age 65 years) were prospectively recruited from 4 hospitals in South Yorkshire between Feb-Dec 2012. All individuals had HRQoL evaluated prior to gastrostomy insertion, with repeated measurements undertaken at 3 months. Assessment was undertaken using EQ-5D, a validated assessment tool and preferential measure used by NICE, producing scores between 0 for dead and 1 for perfect health. Findings were then compared with a separate cohort of population controls ( $n=419)$, to determine if differences existed in HRQoL. Non-parametric statistical analysis was undertaken using a Wilcoxon Rank test to compare longitudinal paired EQ-5D scores, and a Mann-Whitney test to compare EQ-5D scores between groups, with $p$ values $<0.05$ considered significant.

Results 61 gastrostomy patients have been assessed to date. Of these, 3 died prior the 3 month reassessment post insertion. No significant change was shown in mean EQ-5D scores in either the gastrostomy patients $(0.74$ versus $0.73, p=0.11)$ or their carers ( 0.96 versus $0.97, p=0.30$ ) at 3 months following gastrostomy insertion. When compared to population controls, carers had comparable scores to the population controls unlike the gastrostomy patients who had significantly lower mean EQ-5D scores (0.73 versus $0.94, \mathrm{p}<0.0001)$.
Conclusion This study demonstrates that HRQoL does not significantly improve for patients or their carers following gastrostomy insertion. Given that gastrostomy feeding has no positive effect on HRQoL, questions must be raised as to the merits of this intervention if it only serves to improve physiological outcomes.

Disclosure of Interest None Declared.

\section{PWE-184 THE ASSOCIATION OF DIETICIAN FOLLOW UP WITH THE QUALITY OF LIFE IN COELIAC DISEASE}

doi:10.1136/gutjnl-2013-304907.472

1."M A Zubir, ${ }^{2 B}$ Engel. 'Gastroenterology Department, East Sussex Healthcare NHS Trust, Hastings; ${ }^{2}$ Faculty of Health and Medical Sciences, University of Surrey, Guildford, UK

Introduction Coeliac disease (CD) is present in 1\% of the UK population however there are still many undiagnosed cases in the community. The main treatment for this condition is gluten free diet. The role of dieticians in the follow up care of CD patients is crucial especially with regards to educating CD patients about their dietary requirement as well as their role in disease monitoring. The aim of the study is to assess the association of dieticians follow up on the quality of life in CD patients.

Methods CD patients who were identified on histology over a 10 years period in East Sussex were sent a socio-demographic questionnaire and the Coeliac Disease Quality of Life (CD-OOL) survey by post. The participants of the study who attend dietician follow up were then compared with those who did not. Multiple linear and logistic regression analyses were used to identify any significant relationships

Results Overall, 58 participants (34\%) completed and returned the questionnaires. Dietician involvement appeared to be the most important predictor (beta $=-0.424, \mathrm{p}=0.001$ ) for overall CDOOL score but surprisingly, the negative value indicates that dietician involvement was associated with a lower quality of life score. Increasing age seemed to have a positive impact (beta $=0.312$, $p=0.011$ ) on overall quality of life. However, a change of the recent gluten free prescription (beta $=-0.246, p=0.037$ ) which limits the products available and having initially presented with gastrointestinal symptoms (beta $=-0.24, p=0.044$ ) were found to have negative contributions to the overall CDQOL score. The overall model fit was moderate $\left(R^{2}=0.336\right)$. Logistic regression was used to identify the likelihood of dietician follow up and the only significant predictor identified was the overall CDQOL score (OR 0.95, p = 0.019) which indicates that those with higher quality of life score were less likely to have dietician follow up.

Conclusion Dietician follow up is associated with poorer quality of life but due to the nature of this study, cause and effect cannot be established. It may be that patients who have poorer quality of life chose to see a dietician and longitudinal study is required to further assess this association. This study also suggests that the recent change to the gluten free prescription has negatively impacted the quality of life of those with CD. This fact should be taken into consideration for any future health providers dealing with local CD service provisions.

Disclosure of Interest None Declared.

\section{PWE-185 USE OF DEEP SEDATION FOR A PERCUTANEOUS GASTROPEXY SERVICE IN A DISTRICT GENERAL HOSPITAL}

doi:10.1136/gutjnl-2013-304907.473

1."N Siddique, 'C Nunes, ' A F Muller. 'Gastroenterology, Kent and Canterbury Hospital, Canterbury, UK

Introduction All patients with head and neck cancer referred for nutritional support with enteral feeding to our unit undergo direct 